\title{
La participación autonómica y local en redes de cooperación europea ${ }^{1}$
}

\author{
Luis Bouza García \\ Colegio de Europa/Universidad de East Anglia \\ luis.bouza@coleurope.eu \\ Recibido: 27 de abril de 2012 \\ Aceptado: 18 de julio de 2012
}

\begin{abstract}
Resumen
Este artículo analiza la participación de las comunidades autónomas y entidades locales españolas en redes de cooperación con otros gobiernos sub-estatales europeos. El artículo analiza si estas redes constituyen una nueva forma de intervención de las CCAA y entidades locales en los asuntos comunitarios. La primera hipótesis del trabajo es que la participación de los gobiernos sub-estatales españoles en estas redes se explica por la búsqueda de medios de acción complementarios a los mecanismos de intervención más institucionalizada. En segundo lugar se sugiere que la motivación de los gobiernos sub-estatales españoles para participar en dichas redes es la de promover sus intereses mediante la cooperación con actores que comparten sus objetivos. El artículo emplea la metodología del análisis de red para estudiar en qué redes participan los actores españoles y emplea estos resultados para entender su motivación. El artículo concluye que los gobiernos sub-estatales españoles, sobre todo las CCAA, participan intensamente en redes de cooperación en ámbitos de su competencia, lo cual constituye una estrategia de representación de intereses complementaria de la representación institucionalizada ante las instituciones comunitarias.
\end{abstract}

Palabras clave

Gobernanza multinivel, Intereses sub-estatales en la UE, Mecanismos informales, Análisis de red.

\section{The Involvement of Regional and Local Governments in European Cooperation Networks}

\begin{abstract}
This article analyses the participation of Spanish regions and local governments in networks of cooperation with other European sub-national governments. The article asks if these networks are a new form of activism in the EU by Spanish regional and local governments. The first hypothesis is that the participation of Spanish sub-national governments in these networks can be explained as a way to develop ways of influence that can complement more institutionalised venues for the representation of sub-national interests in the EU. The second hypothesis is that the motivation of Spanish sub-national governments to participate in these networks is to promote their own interests by cooperating with other actors sharing the same objectives. The article uses network analysis methodology in order to analyse in which networks Spanish actors participate and uses these results in order to understand their motivation to do so. The article concludes that Spanish sub-national governments, in particular regional ones, participate intensely in networks of cooperation in matters of their own competence and that this constitutes a strategy of interests representation that can complement representation in more institutional venues.
\end{abstract}

Key words

Multilevel Governance, Sub-National Interests in the EU, Informal Mechanisms, Network Analysis.

1 Este estudio se basa en parte en mi participación en el proyecto “The Brussels Offices of the Regions" dirigido por el profesor Justin Greenwood, de la Universidad Robert Gordon de Aberdeen, Reino Unido así como en el periodo en que he sido becario de la subdirección de programas formativos en administración local del INAP. Deseo también agradecer a dos evaluadores anónimos de la REALA sus comentarios constructivos que me han permitido mejorar el artículo. Naturalmente las opiniones expresadas son sólo las mías, al igual que cualquier error que el artículo pudiera contener. 


\section{INTRODUCCIÓN}

Durante más de tres décadas las ciudades y regiones de Europa han desarrollado su presencia ante la Unión Europea. Esta presencia ha tenido una importante manifestación institucional en la creación del Comité de las Regiones y de la posibilidad de que las regiones de Europa participen en las deliberaciones del Consejo cuando se traten asuntos de su competencia. También es de destacar que el activismo de las regiones y entidades locales les ha llevado con frecuencia a defender sus intereses directamente ante las instituciones en Bruselas siendo llamativo el desarrollo de hasta 250 oficinas de representación regional en Bruselas (Greenwood 2011a, Verboven 2011). También es muy significativa la creación de numerosas asociaciones que promueven los intereses de las regiones y los gobiernos locales tales como el Consejo Europeo de Municipios y Regiones (CEMR), el Consejo de Regiones Periféricas y Marítimas de Europa (CPMR), la Asamblea Europea de Regiones (AER) o la organización Eurocities.

Esta tendencia ha coincidido temporalmente con la consolidación del Estado autonómico español. Las comunidades autónomas se han sumado de manera relativamente entusiasta a esta dinámica y han desarrollado su presencia directa ante las instituciones comunitarias. Hasta 2011, año en que se cerraron las oficinas europeas de Asturias y de Castilla la Mancha, todas las CCAA tenían una representación en Bruselas. Esto sitúa a las CCAA muy por encima de la presencia media de las regiones de otros países, puesto que en 2011 sólo una de cada dos regiones europeas disponía de una representación en Bruselas (Verboven 2011: 9). Además todas ellas se han distinguido como actores especialmente importantes en la medida en que a diferencia de buena parte de las otras regiones de Europa éstas ejercen poderes legislativos, lo cual constituye un factor de diferenciación muy importante entre los diferentes actores subestatales (Tuñón Navarro 2008a: 15). En este sentido las CCAA participan en asociaciones de regiones con poderes legislativos como Reg-Leg o de la Conferencia de Asambleas Legislativas Regionales de Europa (CALRE) y pueden participar en el Consejo de la Unión Europea cuando se debatan asuntos relativos a sus competencias. También participan en la formación de la posición del Estado ante las instituciones europeas (la llamada "fase ascendente" del proceso), a través de la Conferencia para asuntos relacionados con la Unión Europea (CARUE) (Castellà Andreu 2008, De Castro Ruano y Ugalde Zubiri 2011).

Sin embargo el balance del activismo local y regional de los últimos 30 años es relativamente escaso. La participación de las regiones a través del Comité de las regiones no ha significado un empoderamiento real de las regiones (Warleigh,2002: 183-185, Greenwood, 2011b: 175, Brunazzo, 2010: 302) debido a "la heterogeneidad de sus miembros de naturaleza tanto local como regional [... ]; la falta de reconocimiento político al no otorgársele el rango de institución quedando marginado como un mero órgano consultivo; la falta de competencias en materias habitualmente gestionadas por las regiones (investigación, industria o agricultura, entre otras); el hecho de que sus dictámenes no sean vinculantes; o el seguimiento de una lógica estatal y no regional en el reparto de sus escaños" (Tuñón Navarro 2008a: 28-29). Greenwood señala que es especialmente significativo el fracaso las regiones en uno de sus principales objetivos, el adquirir un peso específico en la definición de la política europea de cohesión territorial (Greenwood 2011b: 175-178). Esto es especialmente importante para las entidades locales y CCAA españolas debido al impacto de las perspectivas financieras 2014-2020 por las que España se convertiría en un contribuyente neto al presupuesto comunitario (Diputación de Barcelona 2011).

El objetivo de este artículo es investigar si las regiones y entidades locales españolas han adoptado estrategias de influencia complementarias a la participación a través de los mecanismos institucionales mediante estrategias de cooperación informal. Tuñón Navarro señala que "en muchas ocasiones los cauces informales, aquellos a los que la literatura apenas hace vagas referencias, son los más efectivos. Estos cauces más informales, por su propia naturaleza menos fiscalizados, permiten a las regiones una interacción a escala europea mucho más directa que les aporta una aproximación en ocasiones singular, y muchas veces diferenciada." (Tuñón Navarro 2008b: 171-172). Recientemente se ha sugerido que uno de lo mecanismos informales al que las regiones están recurriendo más intensamente en los últimos años es la participación en redes de cooperación especializada con otras regiones (Verboven 2011). A diferencia de los mecanismos de participación institucional, las redes son mecanismos informales. Por otra parte, se diferencian también de las asociaciones señaladas más arriba en que son más especializadas y tienen una naturaleza más difusa.

Este análisis tiene una gran importancia empírica y teórica. A pesar del interés por la participación de los gobiernos territoriales españoles en la gobernanza multinivel europea (Morata 2002, Beltrán 2007, Tuñón Navarro 2008a, Castellà 2008, Rodríguez 2011) no disponemos de un análisis de la participación de las CCAA, y menos todavía de las entidades locales, en este tipo de redes informales. Este análisis también tiene una importancia teórica significativa. La noción de gobernanza, que en la última década ha sido el paradigma dominante para el análisis del proceso de elaboración de políticas públicas europeas, señala que los distintos actores del proceso interactúan de manera no jerárquica e informal. Sin embargo, la práctica política es bien distinta, puesto que los propios mecanismos de gobernanza como el Libro Blanco de la Comisión Europea de 2001 reservan un apartado clara- 
mente diferenciado para las regiones y entidades locales. En este sentido se ha señalado que las organizaciones representativas de las regiones como CEMR, CPMR o Eurocities, rechazaron insistentemente la asimilación de las mismas a los actores privados que intervienen en los procesos de gobernanza y reivindicaron un papel específico durante la elaboración del libro blanco (Sloat 2003). Por lo tanto un posible aumento de la cooperación mediante mecanismos informales aproximaría a los gobiernos sub-estatales a las estrategias de representación funcional y no territorial de la sociedad civil (Smismans 2004; Knodt y Greenwood 2011), lo cual supone una innovación significativa en la actividad de las regiones a escala europea.

Este artículo plantea dos grandes preguntas sobre la participación de las administraciones territoriales españolas en estas redes. En primer lugar es necesario saber si las CCAA y las entidades locales se han sumado a la nueva tendencia de constitución y participación en redes ya mencionada. En segundo lugar resulta también necesario plantearse qué motivaciones tienen dichos gobiernos para participar en estas redes. Para responder a la primera pregunta se realizará un análisis empírico de la presencia de las CCAA, diputaciones y Ayuntamientos españoles en redes europeas, mediante el método del análisis de red. En respuesta a la segunda pregunta se intentará, a la luz de las variables explicativas empleadas por la literatura disponible, explicar las motivaciones de dicha participación mediante el análisis de las diferencias entre el papel de varios actores y de los ámbitos temáticos en que se concentra la participación en las redes.

La siguiente sección examina el funcionamiento y características de las redes de cooperación en que participan los gobiernos sub-estatales así como su significación teórica a la luz del paradigma de la gobernanza multinivel. Se analizan también las diferencias entre mecanismos formales e informales y se exponen las hipótesis de trabajo. La tercera sección presenta el análisis empírico de la presencia de las CCAA y entes locales en estas redes y la cuarta examina las motivaciones para participar en dichas redes a la luz de los elementos del análisis empírico y de la literatura disponible.

\section{EL NIVEL REGIONAL Y LOCAL Y LA INTEGRACIÓN EUROPEA: EL LÍMITE DEL MODELO TRADICIONAL}

El progresivo desarrollo de la participación de los gobiernos sub-estatales en la integración europea ha dado lugar a un número importante de trabajos teóricos y empíricos. Por una parte, en los años de cambio de siglo se acuñó el concepto de gobernanza multinivel (Marks 1996 et al, Rojo Salgado 1999, Comisión Europea 2001, Morata 2002, Warleigh 2002) para significar que el activismo de las regiones es una manifestación de una nueva forma de funcionamiento de la UE. Esta noción sugiere que la elaboración de políticas no es el resultado de una autoridad centralizada que decide por su carácter jerárquicamente superior sino de la interacción y negociación permanente entre distintos niveles de gobierno y actores públicos y privados. En este sentido los actores que intervienen en la elaboración de las políticas públicas ya no son sólo aquellos que tienen capacidad formal de decisión sino todos aquellos que se ven afectados por la misma (Smismans 2004). Por ser unos de los principales receptores de normativa comunitaria, las regiones y municipios de Europa constituyen uno de los actores centrales de este cambio de paradigma. La literatura ha sugerido distintos motivos para el desarrollo de este activismo. La explicación más aceptada es que ante la extensión de las políticas europeas a materias de competencia tradicionalmente regional o local estos actores necesitan estar presentes en la UE puesto que en la misma se deciden asuntos vitales para ellos. Puesto que tradicionalmente el único representante de los Estados miembros ante la UE es el gobierno central, la consecuencia de la no activación de los gobiernos sub-estatales sería que la integración europea pondría en cuestión la distribución constitucional de competencias a escala nacional si el Estado puede decidir en Bruselas sobre materias de competencia regional. En este sentido, la primera explicación es que el activismo de los gobiernos sub-estatales ante la UE es una manifestación lógica de la tradicional tensión competitiva entre distintos niveles de gobierno (Marks et al. 1996; Jefferey 2005; Keating y Hooghe 2006). También se han dado explicaciones relativas al activismo de las propias instituciones europeas. Estas explicaciones sugieren que los gobiernos sub-estatales constituyen un contrapeso al predominio de los Estados (Marks et al 1996, Morata 2002) y que por lo tanto la Comisión tiene un especial interés en asociarlas a las discusiones sobre políticas europeas de la misma manera en que la ha apoyado a las organizaciones de la sociedad civil para introducir demandas o presiones sobre los Estados (Greenwood 2011b).

Uno de los aspectos centrales del paradigma de la gobernanza europea es que "el fomento de las redes de cooperación interadministrativa, la transparencia, la información y los mecanismos de mercado se han convertido en el leitmotiv de la administración comunitaria" (Morata 2002: 8). Sin embargo, a pesar del énfasis de las instituciones y de la doctrina académica en los aspectos informales, negociados y no jerárquicos de la noción de gobernanza tales como la capacidad de influencia sin decisión formal o el recurso a la soft law (Morata 2002), es indudable que las regiones tienen un estatus propio y más institucionalizado que el resto de actores que participan en la elaboración de las políticas comunitarias. Por su naturaleza pública, el Libro Blanco sobre la gobernanza de la Comisión europea (2001) les da a las regiones un trato diferenciado de otros actores que intervienen en el proceso 
de toma de decisiones como las organizaciones de representación de intereses de la sociedad civil. Debido a su condición de autoridades públicas receptoras en buena medida de las normas comunitarias las regiones disponen de una representación propia en el Comité de las Regiones y un trato diferenciado en todos los mecanismos de consulta previa a la toma de decisiones. A modo de ejemplo, sus oficinas de representación están exentas de inscribirse en el Registro de Transparencia a diferencia de los representantes de intereses sectoriales (Greenwood 2011a: 439). Varios autores insisten pues en la necesidad de establecer una distinción entre los distintos mecanismos de influencia empleados por los gobiernos sub-estatales (Keating y Hooghe 2006: 244; Tuñón Navarro 2008b; Brunazzo 2010), en especial en la medida en que las diferencias entre lobbies territoriales y funcionales se difuminan cada vez más (Knodt y Greenwood 2011). Pensamos pues que la distinción entre mecanismos formales e informales de intervención en las políticas europeas es importante desde el punto de vista teórico y empírico sin pretender sin embargo que se trate de una oposición dicotómica, puesto que ambas suelen ser las dos caras de una misma estrategia de influencia de los actores sub-estatales.

Los mecanismos formales de influencia y consulta son aquellos en los que los gobiernos territoriales participan en la elaboración de las políticas precisamente por ser parte del sector público y autoridades democráticas. Mediante el principio de subsidiariedad las instituciones comunitarias reconocen la importancia de los gobiernos sub-estatales en la ejecución de las políticas europeas. Sin embargo este principio también tiene una dimensión ascendente (Tuñón Navarro 2008a: 25-27), en la medida en que las instituciones han reconocido que ante las críticas por la falta de legitimidad democrática de la UE resulta necesario asociar al nivel de gobierno más cercano al ciudadano a la elaboración de las políticas públicas. Precisamente por ello se le da un papel formalizado a las regiones en el proceso de elaboración de políticas públicas, ya sea a nivel consultativo en el Comité y otros foros o incluso como representantes de sus Estados en el Consejo cuando se debaten asuntos de su competencia. De esta manera los gobiernos territoriales actúan como poderes públicos en la formulación de la agenda e incluso en la toma de decisiones puesto que en el caso de Estados muy descentralizados como Bélgica, las regiones pueden llegar a sustituir al Gobierno en las votaciones en el Consejo. Resulta importante señalar que existe una diferencia fundamental entre las regiones y las entidades locales. La institucionalización del papel de las regiones es mucho más importante que el de los entes locales: los gobiernos locales tienen una presencia reducida en el Comité de Regiones (en el caso español, 4 miembros propuestos por la FEMP frente a los 17 representantes de las CCAA), no pueden participar en el Consejo de Ministros y tienen poca presencia directa en Bruselas.

Por otra parte, las regiones también tratan de influenciar el proceso de toma de decisiones como partes interesadas en el mismo. De este modo las regiones ya no participan mediante mecanismos formalmente establecidos en el marco de un contexto de democracia representativa, sino más bien en un contexto más cercano al concepto más informal de gobernanza como "gobierno sin gobierno" en el que el papel de los participantes en la elaboración de políticas no depende de su representatividad territorial sino de su capacidad funcional para representar un determinado interés sectorial (Smismans 2004; Greenwood 2011a: 438). Por lo tanto los mecanismos informales aproximan a los gobiernos sub-estatales a las formas de acción colectiva de los actores de la sociedad civil (Rodríguez 2011, Knodt y Greenwood 2011). De esta manera, las posibilidades de influencia de las regiones y entes locales no radican en un derecho a participar en el proceso de toma de decisiones sino más bien en su capacidad de aportar inputs de los que carecen las instituciones comunitarias, ya sea una experiencia concreta de de un ámbito de gobierno, capacidad de desarrollo de una política o apoyo político.

Por lo tanto esta lógica no remite tanto a los aspectos formales de la estrategia de gobernanza multinivel de la Unión Europea como la coordinación entre los distintos niveles de gobierno, sino más bien a los mecanismos mediante los que los intereses sectoriales intervienen en las políticas públicas. Las aproximaciones jurídicas al papel de los gobiernos sub-estatales en la UE (Castellá Andreu 2008) tienden a obviar la importancia de la cooperación informal (Tuñón Navarro 2008b). Este tipo de activismo basado en estrategias de influencia mediante el convencimiento y la articulación de coaliciones de actores de distintos ámbitos remite a nociones clásicas como las comunidades epistémicas (Haas 1992), las coaliciones promotoras (Sabatier y Jenkins-Smith 1993) o el establecimiento de la agenda política e institucional (Kingdon 2003). La literatura señala la creciente importancia de este tipo de comunidades en el ejercicio de influencia sobre las instituciones comunitarias (Quittkat y Kotzian, 2011). Este artículo examina en mayor detalle la participación de los gobiernos subestatales españoles en una de las manifestaciones más significativas en los últimos tiempos del uso de nuevos canales de intervención, las redes de cooperación (Verboven 2011).

La literatura ha sugerido distintas explicaciones para el auge de estas formas de cooperación informal. En este sentido es tradicional señalar que las regiones con mayor grado de autonomía o una identidad más diferenciada respecto a su Estado de pertenencia están más activas en Bruselas y desarrollan por lo tanto un activismo más intenso (Keating y Hooghe 2006). Por otra parte, también se sugerido la importancia de factores económicos. 
En este sentido, no sería tanto una cuestión jurídica o político-identitaria la que mueve a las regiones a activarse a escala europea sino la importancia del interés material en juego y la capacidad económica de las regiones implicadas para articular distintos tipos de mecanismos (Verboven 2011). Por último, también se han dado explicaciones más institucionalistas que sugieren que los factores decisivos son la antigüedad, la capacidad y el activismo de las representaciones regionales en Bruselas, que pueden desarrollar una búsqueda de autonomía respecto a su gobierno regional parecida a la de su región respecto a su Estado (Greenwood, 2011a).

Si bien todas estas dimensiones se tendrán en cuenta en la sección que tratará de explicar la participación de gobiernos sub-estatales españoles en dichas redes, la hipótesis de este trabajo es que la explicación más pertinente para este auge de la cooperación en red tiene que ver con la insuficiencia de los mecanismos de representación institucionalizada (Tuñón Navarro 2008a, Greenwood 2011a). Evidentemente esta afirmación debe ser matizada: los gobiernos sub-estatales no han renunciado en modo alguno a la cooperación formalizada. De este modo resulta interesante recordar que la cooperación informal fue la estrategia adoptada por los gobiernos sub-estatales cuando los tratados no permitían todavía una participación más intensa de las regiones y entes locales a través del Comité de las regiones o su participación en el Consejo (Morata 2002: 9). Al contrario, el disponer de un papel institucionalizado y reconocido por las instituciones es una ventaja que tratan de obtener el resto de actores que intervienen en la elaboración de políticas públicas, como por ejemplo la sociedad civil organizada, puesto que permite obtener una mayor atención por parte de las instituciones (Quittkat y Kotzian 2011). Por lo tanto la cooperación informal mediante la constitución de redes no es una alternativa sino un complemento de los mecanismos formales de representación. Resulta por lo tanto pertinente interrogarse sobre qué aportan las redes a la estrategia de influencia regional. Este tipo de redes tienen al menos tres grandes características innovadoras. En primer lugar uno de los aspectos fundamentales de las redes es su dimensión temática. Es frecuente que se articulen en torno a un ámbito o una política pública en la todos los miembros tienen interés y un alto nivel de experiencia. Las redes no funcionan como norma general de manera jerárquica, es decir en base a la naturaleza de actores públicos de sus miembros, sino más bien en torno a una especialización técnica de sus miembros en torno a una política de interés común (Castro Ruano y Ugalde Zubiri 2011: 90). Este es claramente un aspecto en el que las redes permiten una activación más eficiente que los mecanismos formales, puesto que permiten cooperar y tratar de obtener influencia en aquellos asuntos excluidos de las competencias del Comité de las Regiones pero de gran importancia para los gobiernos sub-estatales tales como la investigación, la industria o la agricultura (Tuñón Navarro 2008a: 28-29). En este sentido comparten una característica con las organizaciones de la sociedad civil que es la de participar en la política comunitaria en base a su experiencia y capacidad de aportar propuestas, por lo que no es raro por lo tanto que las redes se caractericen por una importante apertura a dichas organizaciones (Greenwood, 2011a). En segundo lugar las redes constituyen formas de cooperación y de influencia menos institucionalizadas que las asociaciones de regiones y municipios pues cuentan con una presidencia rotatoria y un personal propio reducido o inexistente (Greenwood 2011a). En este sentido las redes y mecanismos de cooperación informal también permiten acercarse a otros actores que intervienen en el proceso de decisión, permitiendo establecer alianzas en torno a propuestas y políticas concretas (Greenwood 2011a, Verboven 2011). Mientras que en los mecanismos formales los gobiernos territoriales son el eslabón más débil de cualquier posible coalición con los Estados o las instituciones, en un esquema más informal, una red de gobiernos regionales puede ser el líder de una coalición de la sociedad civil y otros actores sectoriales, precisamente debido a su capacidad de establecer puentes hacia las instituciones mediante mecanismos formales.

En tercer lugar a diferencia mecanismos como la participación en la formación de la posición del Estado en la CARUE, la participación en el Consejo o de la cooperación transfronteriza más formalizada (Beltrán 2007), la participación en redes no impone la obligación de coordinación con el gobierno central sino que permite el desarrollo de agendas propias entre niveles territoriales de distintos Estados con autonomía respecto a los mismos (Jefferey 2005).

Tras esta aproximación teórica a los distintos mecanismos de intervención de los gobiernos sub-estatales ante la Unión Europea, presentamos de manera sucinta las hipótesis que vamos a examinar respecto a la participación de las CCAA y entidades En este sentido la constitución de redes permite sortear bloqueos y resistencias internas puesto los mecanismos de participación formal requieren en general un fuerte consenso entre las regiones interesadas y un acuerdo del Estado en este sentido y ninguno de estos supuestos está garantizado (Castellà Andreu 2008: 87). Por lo tanto la cooperación informal permite defender posturas o intereses que no suscitan la unanimidad a nivel nacional. Por ejemplo redes como Metrex o Cities for Cohesion desarrollan acciones de lobby para tratar de dar más peso a la agenda de cohesión territorial en detrimento de la agrícola independientemente de la agenda de los Estados.

Tras esta aproximación teórica a los distintos mecanismos de intervención de los gobiernos sub-estatales ante la Unión Europea, presentamos de manera sucinta las hipótesis que vamos a examinar respecto a la partici- 
pación de las CCAA y entidades locales en redes de cooperación europea y respecto a su motivación. La primera hipótesis es que las administraciones españolas han adoptado esta estrategia de manera complementaria a su papel actual, a consecuencia de los límites del activismo más institucionalizado pero no como alternativa al mismo. Esto puede manifestarse de varias maneras. En primer lugar, podemos esperar una fuerte concentración de las redes temáticas en los aspectos mencionados anteriormente como de interés regional o local pero ausentes de las competencias del Comité de las regiones, tales como la agricultura o la industria. En segundo lugar, también podemos esperar una concentración en ámbitos de mayor activismo de la sociedad civil, puesto que ya se ha señalado que esta estrategia puede contribuir a establecer coaliciones con este tipo de actores. La tendencia contraria, es decir, que la participación en redes sea una alternativa en lugar de un complemento a estrategias más formales, tendría como manifestación principal que las entidades más activas en la cooperación en red fuesen aquellas con menor presencia institucional, como por ejemplo las entidades locales. En cuanto a las motivaciones del uso de esta estrategia Greenwood (2011a) señala que las redes contribuyen a fomentar la influencia de los gobiernos subregionales y a evitar el coste de una representación directa. Nuestra segunda hipótesis es que la motivación principal de los gobiernos sub-estatales españoles consiste en obtener influencia, puesto que hemos asumido más arriba que esta estrategia no es una alternativa sino un complemento a la infraestructura de representación en Bruselas de las que ya disponen los gobiernos territoriales españoles.

Desde el punto de vista metodológico la caracterización de las redes como formas de cooperación menos institucionalizada y por tanto más fluidas e inestables dificulta su estudio y análisis. En este sentido es difícil estar seguro de cubrir el conjunto de redes existentes, así como conocer sus miembros. En términos prácticos resulta difícil trazar una línea que distinga claramente entre redes y asociaciones, puesto que muchas redes terminan institucionalizándose, de acuerdo con la tendencia de cualquier organización a perpetuarse. Por lo tanto este análisis tomará como principal criterio de distinción el de redes u organizaciones de cooperación específica, es decir, aquellas en las que no participan todos los poderes territoriales de Europa sino aquellos agrupados por intereses o causas comunes. En este sentido no se han considerado algunas de principales asociaciones de gobiernos territoriales europeos, la AER, CEMR, la CPMR o Eurocities (véase Greenwood 2011a para un criterio similar). Tampoco se han tenido en cuenta redes como CEPLI o CEMR en la que los miembros no son los gobiernos a título individual sino una representación centralizada como la FEMP.

El análisis empírico se fundamenta en un estudio de la participación de las CCAA y entidades locales españolas en redes transnacionales europeas. Por una parte, la dificultad metodológica de esta tarea es la que siempre se plantea cuando se analiza la integración europea desde una perspectiva descentralizada: la diversidad inherente a formas de cooperación abierta a organizaciones provenientes de toda Europa (Rodríguez 2011:7; Verboven 2011: 4). Además como ya hemos dicho muchas de ellas son informales y fluidas, por lo que no es raro que cambien frecuentemente de denominación y de miembros, por lo que la información disponible es siempre fragmentaria. Para hacer frente a dicho desafío hemos cruzado los datos de distintas fuentes para asegurarnos una mayor fiabilidad. Por una parte se recopilan los datos disponibles en los anuarios del Comité de las Regiones y de la Dirección General de política regional de la Comisión europea. Por otra parte se compila una lista de las redes transnacionales en que participan las CCAA y las entidades locales españolas en base a sus informes relativos a su actuación internacional, tal y como la Guía de redes europeas de la Diputación de Barcelona² o el Informe de la Secretaría General de Acción Exterior del Gobierno Vasco (Castro Ruano y Ugalde Zubiri 2011). Una vez compiladas dichas listas se verifica la participación de al menos una Comunidad Autónoma o entidad local en la misma al mismo tiempo que algún otro socio europeo. El resultado es una lista de 52 redes europeas con participación de al menos una de las 74 entidades españolas incluidas en el análisis (véanse las tablas en el anexo). Dicha verificación se ha realizado visitando la página web de cada una de estas redes y comprobando qué entidades españolas aparecen como miembros de la misma. Evidentemente este método tiene la desventaja de que no se tienen en cuenta redes más informales o que simplemente no dispongan de página web. Sin embargo, esto puede ser una ventaja desde el punto de vista del análisis, en la medida en que se centra en las redes mejor establecidas, lo que indica que se mantienen relativamente activas. Por otra parte no es seguro que dichas redes mantengan absolutamente actualizada su lista de miembros. Sin embargo, esta desventaja se compensa parcialmente porque debido al gran número de redes y entidades consideradas el resultado agregado puede considerarse digno de confianza.

Estos datos han sido estudiados de manera fundamentalmente cuantitativa. En primer lugar se realiza un estudio descriptivo del número de actores españoles que participan en cada red, así como los sectores temáticos de cooperación. Seguidamente se realiza un análisis de red centrado en el estudio de la centralidad individual de los actores, es decir, qué papel desempeña cada administración respecto al conjunto de los actores considerados en términos de proximidad a cada miembro de las redes. Este enfoque permite tener una buena visión de conjun-

2 http://www.diba.cat/web/ri/guia_xarxes_europees 
to tanto del activismo de los gobiernos sub-nacionales españoles como de la importancia relativa de cada uno de ellos en las mismas.

\section{ANÁLISIS EMPÍRICO SOBRE LA PRESENCIA DE LAS CCAA Y LAS ENTIDADES LOCALES EN LAS REDES EUROPEAS}

A través de un análisis de red se ha analizado la presencia de CCAA y diputaciones españolas en redes europeas. Se analizará qué regiones o entidades locales participan más intensamente y cuáles son las redes preferidas de las regiones españolas.

En primer lugar, el estudio incluye a las 17 CCAA y las 2 ciudades autónomas, 22 diputaciones o administraciones insulares y 33 ayuntamientos. La tabla número 1 más abajo señala claramente el papel predominante de las CCAA, tanto por su número (todas ellas participan en alguna red) como por el número de redes en que participan, puesto que como media cada comunidad autónoma participa casi en 10 redes, mientras que los ayuntamientos sólo lo hacen en algo más de dos y las diputaciones no alcanzan esa cifra. Estas cifras señalan que las CCAA españolas son mucho más activas que la media de las regiones europeas, que participan en una media de 4,84 redes (Verboven 2011:8) mientras que los ayuntamientos y diputaciones se encuentran en un nivel bastante inferior al de dichas regiones. Por otra parte es necesario considerar el tema de cooperación de las redes, es decir, sobre qué tipo de política actúan. Este ese el objeto de la tabla número 2.

TABLA I

Presencia de administraciones españolas en redes europeas

\begin{tabular}{|l|l|l|}
\hline & Número & Número de redes en que participan (promedio) \\
\hline Ayuntamientos & 33 & 2,27 \\
\hline CCAA & 19 & 9,52 \\
\hline Diputaciones & 22 & 1,77 \\
\hline
\end{tabular}

TABLA 2

Pertenencia a redes por ámbito temático

\begin{tabular}{l|c|c|c|}
\hline \multicolumn{1}{|c|}{ Ámbito temático } & $\begin{array}{c}\text { Miembros } \\
\text { españoles }\end{array}$ & $\begin{array}{c}\text { Número } \\
\text { de redes }\end{array}$ & $\begin{array}{c}\text { Número medio } \\
\text { de miembros }\end{array}$ \\
\hline Medio Ambiente sostenibilidad & 46 & 10 & 4,6 \\
\hline Cooperación transfronteriza territorial & 60 & 10 & 6 \\
\hline I+D+I Educación & 55 & 8 & 6,88 \\
\hline Cultura deporte & 21 & 5 & 4,2 \\
\hline Sanidad & 14 & 4 & 3,5 \\
\hline Asuntos sociales & 17 & 4 & 4,25 \\
\hline Regiones con poder legislativo & 28 & 3 & 9,33 \\
\hline Regiones industriales & 11 & 3 & 3,66 \\
\hline Transporte & 12 & 2 & 6 \\
\hline Agricultura & 21 & 2 & 10,5 \\
\hline Inmigración & 7 & 1 & 7 \\
\hline
\end{tabular}

La tabla número 2 sugiere que las redes en que participan los gobiernos locales y autonómicos españoles son fundamentalmente de tres tipos. En primer lugar, cabe destacar formas más o menos tradicionales de cooperación transfronteriza y territorial (Beltrán 2007). En este primer grupo hemos incluido también las redes que agrupan gobiernos locales según criterios geográficos (islas, zona mediterránea y atlántica por ejemplo). Aunque algunas de estas redes son más novedosas se desprenden dos grandes tipos. En primer lugar existen las coope- 
raciones entre regiones fronterizas, que no consisten fundamentalmente en una cooperación reticular sino más bien en eliminar las barreras a la cooperación impuestas por las fronteras estatales. Este tipo de cooperación ha contado frecuentemente con financiación del presupuesto de la política regional europea a través del programa Interreg o los marcos de cooperación interterritorial (Castro Ruano y Ugalde Zubiri 2011: 141). El segundo tipo de organización son las que tratan de representar ante las instituciones de la UE los intereses de los territorios con problemáticas similares debido a su situación geográfica. Mención aparte merecen las agrupaciones de gobiernos, fundamentalmente CCAA, con poder legislativo. Si bien estas redes no son numerosas, está claro que las CCAA españolas están fuertemente representadas en las mismas, puesto que ofrecen una importante oportunidad de diferenciación para las regiones con capacidad legislativa respecto a las que carecen de la misma. En este sentido también puede destacarse las redes culturales, que siempre han tenido un papel importante en la "diplomacia municipal" (Diputación de Barcelona 2008) y en la política de hermanamientos.

En segundo lugar existen redes estructuradas en torno a regiones con intereses económicos compartidos, tal y como pueden ser las agrupaciones de territorios industriales -industrias químicas, del automóvil o textiles por ejemplo y agrícolas- regiones vinícolas, hortofrutícolas.

En tercer lugar existen redes de cooperación en torno a políticas concretas. Si bien muchas de ellas tienen una innegable dimensión económica, en especial aquellas relacionadas con el transporte, la sostenibilidad y la política de investigación e innovación, se diferencian del grupo anterior en que su objetivo no es, en principio, la representación de un determinado tipo de interés, sino una estrategia de cooperación en torno a una determinada política. Estas redes resultan especialmente interesantes no sólo por su número y la cantidad de miembros sino porque se estructuran alrededor de las políticas temáticas de la UE más allá de la política de cohesión territorial, tal y como la política de medio ambiente, sanidad y protección de los consumidores, así como las estrategias de cooperación en materia de innovación y asuntos sociales. Resulta interesante constatar el número de redes relativas a educación e I+D+i y el número de miembros relativamente elevado de cada una de ellas $(6,88)$, lo que sugiere la importancia de las redes en ámbitos con fuerte dinamismo y donde el conocimiento y la promoción de buenas prácticas resultan importantes.

\section{GRÁFICO I}

Representación de la red

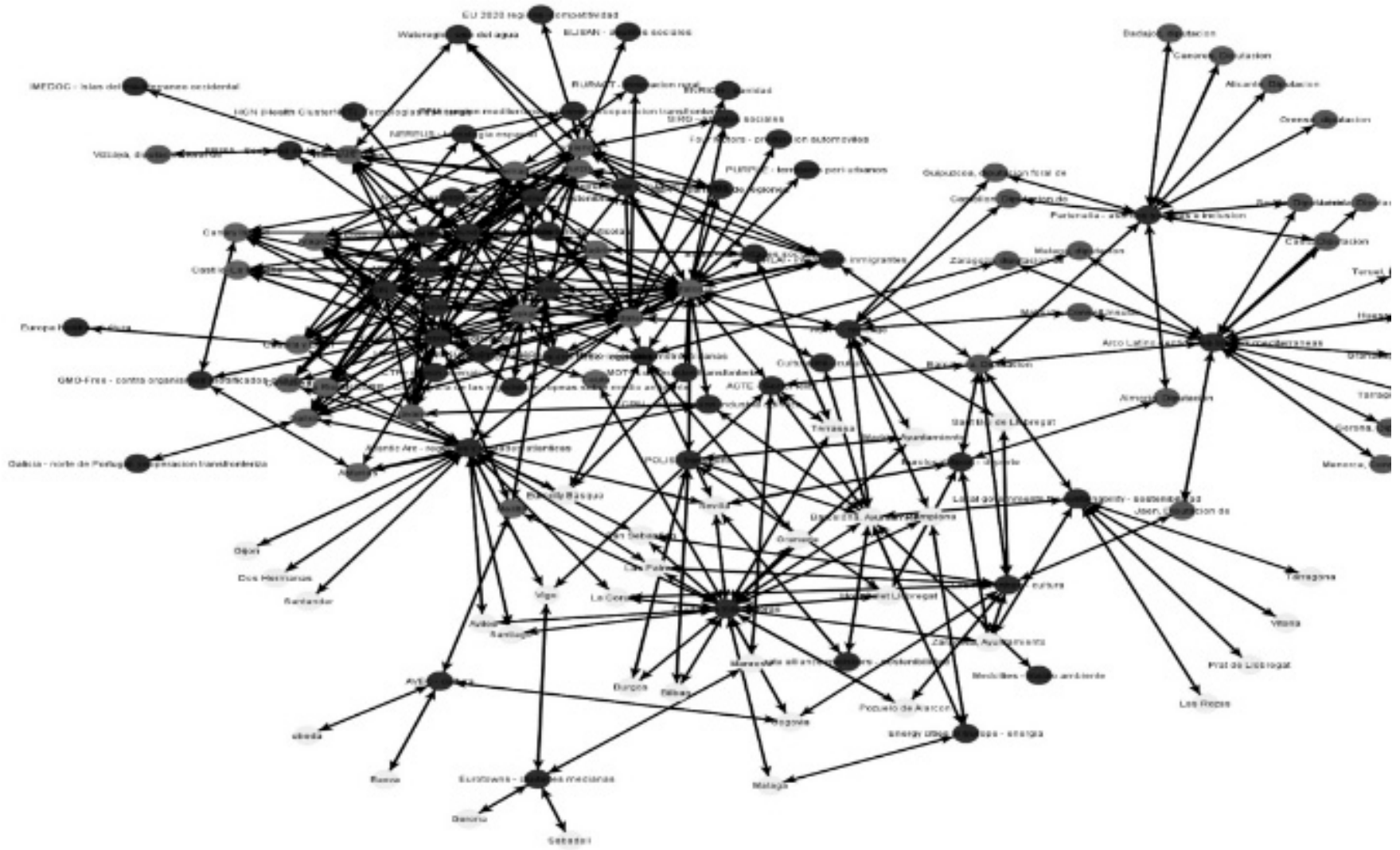

Una vez presentado el marco común sobre el número de actores y las áreas en las que se concentran, es necesario analizar concretamente qué actores y qué redes resultan especialmente importantes. Tal y como hemos señalado más arriba la metodología de análisis de red resulta útil para este estudio. El gráfico número 1 representa 
el conjunto de la red de los gobiernos territoriales españoles en Europa. Los nodos azules son las redes europeas, cuyas relaciones entre sí no están representadas, los rojos las CCAA, los verdes las diputaciones y los amarillos los ayuntamientos.

La ilustración señala claramente que la participación de las CCAA en redes europeas es mucho más intensa que la de los otros actores. También se ve claramente que la principal diferencia entre las diputaciones y los Ayuntamientos no estriba sólo en el número de redes en que participan (tabla 1) sino en la diversidad de las mismas, en la medida que la participación de los Ayuntamientos está mucho más diversificada entre distintas redes, mientras que las diputaciones se concentran fundamentalmente en dos redes, Partenalia y el Arco Latino.

Además de la fuerte diferenciación por el número de redes en que participan, los gobiernos locales y regionales están también fuertemente diferenciados en función del tipo de red en la que participan. Esta constatación es coherente con la literatura que señala que distintos tipos de redes implican a distintos tipos de actores (Verboven 2011). En este sentido, las CCAA tienen una fuerte presencia en redes de cooperación territorial, de medio ambiente, de educación e innovación, sanidad y asuntos sociales, así como de entidades con capacidad legislativa, mientras que los Ayuntamientos se concentran fundamentalmente en redes de cooperación cultural y de medio ambiente y sostenibilidad. Las diputaciones se concentran casi en exclusiva en redes de cooperación territorial como Partenalia y el Arco Latino cuyo objetivo es defender la importancia del nivel territorial intermedio en la UE (Asamblea General de Arco Latino 2012), si bien la red Partenalia también pretende fomentar la cooperación innovadora. En este sentido cabe deducir que la distribución de competencias a escala nacional se reproduce a nivel europeo mediante la selección de las redes en las que participa cada tipo de gobierno sub-estatal. El papel preponderante de las CCAA se explica por lo tanto porque el nivel regional es el que ha visto un mayor solapamiento entre sus competencias y las políticas europeas (Jefferey 2005).

Tras haber descrito más arriba los temas que concentran la atención de los gobiernos sub-estatales españoles, es importante estudiar un aspecto más cualitativo de las mismas mediante medidas de centralidad. Este enfoque sirve para medir la importancia de un actor en una red no sólo por su número de contactos sino también por su posición relativa en el conjunto de la red. En este sentido las organizaciones más centrales son aquellas que están mejor conectadas de manera directa o mediante los caminos indirectos más cortos, al resto d miembros de la red. Realizamos este análisis mediante mediciones de intermediación ("betweennes centrality”) y de cercanía ("closeness centrality"), reflejados en la tabla 3 para las 8 redes más centrales.

TABLA 3

\section{Centralidad de redes europeas}

\begin{tabular}{|l|l|l|l|}
\hline Red & Intermediación & Red & Cercanía \\
\hline Arco Atlántico & 0.16 & ACR+ & 0.37 \\
\hline Arco Latino & 0.13 & ERLAI & 0.37 \\
\hline ACR+ & 0.13 & ACTE & 0.36 \\
\hline Ciudades Educadoras & 0.12 & CALRE & 0.36 \\
\hline CALRE & 0.09 & ArCo Atlántico & 0.35 \\
\hline ERLAI & 0.09 & METREX & 0.34 \\
\hline Partenalia & 0.08 & AREV & 0.34 \\
\hline ACTE & 0.08 & ERRIN I+D & 0.33 \\
\hline
\end{tabular}

Se deduce de la tabla tres que las dos medidas de centralidad coinciden en señalar al mismo tipo de redes. A pesar de lo difícil de crear categorías comunes para un grupo pequeño de redes distintas, es posible señalar dos grandes tipos de organizaciones. Por una parte, se trata de redes temáticas sobre medio ambiente y sostenibilidad y educación y cultura. Por otra parte, el resto son redes de intercambio de experiencias y redes fundamentadas en intereses compartidos, ya sea por sus características geográficas o por su estructura económica.

Es especialmente interesante señalar la existencia de algunas redes en las que participan actores de naturaleza distinta, puesto que son casos que se escapan de la norma tal y como se ha señalado más arriba. El análisis de red confirma claramente la importancia de estas redes pues presentan el mayor índice de intermediación (tabla 3), es decir, aquellas que ponen en relación a actores que de otro modo estarían aislados. En el caso por ejemplo de las redes ACR+ (reciclaje), Metrex (regiones metropolitanas), Polis (transporte) o dos de las redes de carácter 
industrial, ACTE (sector textil) y el CERN (industria química) participan al menos dos tipos de actores e incluso los tres. Tal y como se puede apreciar en el gráfico 1, esto es relativamente poco frecuente puesto que los actores similares tienden a cooperar entre sí en el seno de una red.

Resulta también interesante constatar que estas redes no son necesariamente aquellas que presentan un mayor número de miembros. La tabla 4 más abajo recoge las redes que presentan un mayor grado, es decir, un mayor numero de miembros. Si bien la mayor parte de ellas también están entre las más centrales, véase más arriba, resulta interesante que las dos redes con más miembros (Ciudades Educadoras y CALRE) no ocupen una posición relativa tan importante. Ello se debe a su exclusividad puesto que al ser todos sus miembros del mismo tipo (Ayuntamientos y CCAA) la mayor parte de sus miembros participan en otras redes y por lo tanto estas redes no son tan decisivas a la hora de facilitar la cooperación entre actores.

\section{TABLA 4}

Redes con mayor número de miembros

\begin{tabular}{|l|l|}
\hline Red & Grado \\
\hline Ciudades Educadoras & 0.31 \\
\hline CALRE & 0.30 \\
\hline Arco Atlántico & 0.29 \\
\hline AREV & 0.24 \\
\hline Arco Latino & 0.22 \\
\hline ERRIN I+D & 0.20 \\
\hline ACR+ & 0.16 \\
\hline Partenalia & 0.16 \\
\hline
\end{tabular}

Tras examinar los datos relativos a las redes, es necesario examinar el papel de cada uno de los actores en comparación con el conjunto. El análisis de red confirma la preeminencia de las CCAA señalada por su presencia mucho más importante en redes europeas (tabla 1). Cuando se examina el grado (número de contactos) y la centralidad mediante medidas de cercanía (tabla número 5 más abajo) en este caso las medidas de intermediación es poco útil puesto que no tiene mucho sentido asumir que los distintos niveles de gobierno no tienen contactos más allá de esta red, de las entidades territoriales españolas aparece claramente que son las comunidades autónomas las que desempeñan el papel principal.

TABLA 5

Papel de los actores en el conjunto de la red

\begin{tabular}{|l|c|l|c|}
\hline \multicolumn{1}{|c|}{$\begin{array}{c}\text { Gobi- } \\
\text { erno }\end{array}$} & Grado & \multicolumn{1}{|c|}{$\begin{array}{c}\text { Gobi- } \\
\text { erno }\end{array}$} & $\begin{array}{c}\text { Cer- } \\
\text { canía }\end{array}$ \\
\hline Cataluña & 0.44 & Cataluña & 0.39 \\
\hline Andalucía & 0.27 & Andalucía & 0.37 \\
\hline Euskadi & 0.25 & Euskadi & 0.35 \\
\hline Comunidad Valenciana & 0.22 & Diputación de Barcelona & 0.35 \\
\hline Aragón & 0.19 & Sevilla & 0.33 \\
\hline Ayuntamiento de Barcelona & 0.16 & Ayuntamiento de Barcelona & 0.33 \\
\hline Islas Baleares & 0.16 & La Coruña & 0.32 \\
\hline Murcia & 0.16 & Las Palmas & 0.32 \\
\hline Extremadura & 0.14 & San Sebastián & 0.32 \\
\hline Castilla y León & 0.13 & Comunidad Valenciana & 0.32 \\
\hline
\end{tabular}


Se deduce rápidamente que las CCAA que tienen un papel más activo son "comunidades históricas" o aqueIlas que accedieron antes al máximo nivel competencial. También resulta importante señalar que todas las todas las CCAA de dicha lista disponen de una representación en Bruselas.

Resulta interesante comparar ambas medidas. La columna de la izquierda confirma que la única excepción a la presencia en redes de las CCAA es la del Ayuntamiento de Barcelona. Por otra parte la columna de la derecha muestra una perspectiva bastante distinta, puesto que los ayuntamientos de Sevilla, Barcelona, La Coruña, Las Palmas y San Sebastián están entre las más cercanas al resto de miembros. Esto no significa que tengan un papel más importante que algunas CCAA, sino que están más cerca de otros actores que algunas de ellas. Esto se entiende mejor analizando nuevamente el gráfico 1 en el que se observa que la mayor parte de Ayuntamientos participan en las mismas redes. En este sentido los Ayuntamientos presentes en mayor número de redes, y en especial aquellos presentes en redes en que participan otros actores, están mejor conectados. Nuevamente, esto no debe interpretarse en sentido estricto, asumimos que cualquiera de los actores españoles de la red puede coordinarse directamente con los otros, pero sí que significa que tienen un cierto liderazgo entre el resto de Ayuntamientos, en la medida en que pueden contribuir a difundir información y facilitar contactos entre los Ayuntamientos y otro tipo de redes.

Mención aparte merece la diputación de Barcelona. Sobresale a nivel cuantitativo como uno de los actores más centrales y con participación en más redes (7), pero también cualitativamente a dos niveles significativos. En primer lugar, por su papel en la coordinación de 2 redes con una importante presencia de Diputaciones españolas, Partenalia y Arco Latino. Estas dos redes tienen la especificidad de dirigirse especialmente a los gobiernos intermedios entre la región y el Ayuntamiento y catalizan casi en exclusiva la participación de las diputaciones españolas en redes europeas, en tanto en cuanto que 14 diputaciones, especialmente de la costa mediterránea, sólo participan en estas dos redes. Sin la existencia de estas dos redes coordinadas por la Diputación de Barcelona dichas diputaciones no participarían en ninguna red europea, lo cual convierte a la Diputación de Barcelona en un actor clave. Por otra parte, merece la pena señalar la significativa presencia de municipios Barceloneses en redes europeas (Rodríguez 2011). Barcelona, L'Hospitalet, Manresa, El Prat de Llobregat, Sabadell, Sant Boi y Terrassa participan en distintas redes europeas (véase la tabla 8 en el anexo), que tratan desde el transporte hasta la cultura pasando por la defensa del sector textil. El activismo de estos municipios, 6 de los cuales se encuentran entre los 10 más activos en proyectos europeos en la provincia de Barcelona (Rodríguez 2011:10), se corresponde con la descripción de la Red de Municipios que realiza la diputación de Barcelona: "como el núcleo más activo de dicha red, constituido por los municipios activos en la cooperación europea” (Diputación de Barcelona 2008:20). Por lo tanto la diputación de Barcelona no sólo desempeña un papel importante mediante su presencia en redes, incluyendo la coordinación de las mismas, sino que es un importante catalizador de la participación en redes europeas de otras diputaciones europeas y de los municipios de su provincia.

\section{LAS MOTIVACIONES DE LAS CCAA Y ENTIDADES LOCALES ESPAÑOLAS PARA PARTICIPAR EN REDES EUROPEAS}

Se desprenden varias conclusiones del análisis de las redes. En primer lugar la tabla número 1 señala que las redes con mayor número de miembros españoles son de tipo relativamente tradicional, en el sentido que se centran en cuestiones relativas a la agricultura y la cooperación de gobiernos con potestades legislativas. Por lo tanto resulta interesante constatar que estas redes se concentran en dos ámbitos que suponen una debilidad del Comité de las Regiones: los asuntos agrícolas y la heterogeneidad de dicha institución que rechazan constantemente las regiones con poderes legislativos (Tuñón Navarro: 2008a). Sin embargo, conviene destacar que estas redes agrupan a muchos miembros españoles pero que no son el tipo de cooperación en red más frecuente para los gobiernos sub-estatales españoles. En este sentido se constata que es en los campos del medio ambiente y la sostenibilidad, la I+D+l y la educación (todos ellos ámbitos de competencia regional y local incluidos entre las competencias del Comité de las Regiones) así como la cooperación transfronteriza y territorial donde existen un mayor número de redes y una mayor participación de gobiernos sub-estatales españoles en números absolutos. Además estas políticas tienen dos características destacables. Por una parte son propicias a la cooperación en red puesto que tienen un alto potencial de innovación en ámbitos en que existe un fuerte activismo por parte de la sociedad civil organizada a nivel comunitario (Greenwood 2011a). Por otra parte son ámbitos en los que las autoridades regionales y locales tienen importantes competencias. Esto sugiere que para estos gobiernos las redes informales constituyen foros de cooperación sobre el impacto de la UE en su ámbito competencial. Se ha señalado además que cada tipo de nivel territorial tiene un perfil distintivo, en la medida que el las CCAA, diputaciones y ayuntamientos participan en redes diferentes, al igual que difieren por la intensidad de su participación. Precisamente por ello resulta interesante haber constatado que las redes más centrales son aquellas que agrupan a distintos niveles de gobierno. Éstas son fundamentalmente redes relativas al medio ambiente y de defensa de intereses geográficos o económicos. Esto significa que estas son las redes con mayor probabilidad de tener contacto con gobiernos territoriales españoles. 
Este análisis permite por lo tanto adelantar una expectativa sobre el tipo de motivación para participar en estas redes. Dos motivaciones aparecen de manera sobresaliente. En primer lugar la defensa de intereses particulares que comparte características de tipo geográfico o económico. Esto constituye una manifestación importante del activismo de los gobiernos sub-estatales, puesto que muestran una disposición no sólo a participar mediante los canales establecidos sino a crear organizaciones que permitan presionar a favor de sus intereses de manera informal pero efectiva. Ante el impacto relativamente escaso de la presencia formal, los poderes regionales y locales parecen activarse en colaboración con otras organizaciones para tratar de influenciar directamente en los asuntos de su interés. Nos encontramos por lo tanto en una lógica de influencia en la que los gobiernos territoriales actúan para defender sus intereses. En este sentido la diputación de Barcelona pone en valor la importancia de estas redes para defender sus posiciones en varias políticas relevantes:

"Hemos utilizado todas las plataformas europeas en las que tenemos presencia (Arco Latino, CEPLI, CMRE, Cities for Cohesion...) para acercar a las instituciones comunitarias la realidad, los intereses y las necesidades de nuestro territorio y de sus ciudadanos. En este sentido hemos participado en debates tan transcendentes para la UE como la definición de la Estrategia 2020, la definición de la política de cohesión, el lanzamiento del Pacto de Alcaldes contra el cambio climático, la Directiva de Servicios, el Libro Verde sobre el futuro de la Política de Cooperación al Desarrollo o la creación del ARLEM en el marco de la Unión para el Mediterráneo." (Diputación de Barcelona 2011: 6)

En segundo lugar, la defensa a nivel europeo de políticas de interés regional o local como el medio ambiente y la educación también resultan relevantes. Esta constatación va en la misma línea que los análisis que señalan que la principal motivación del activismo de los gobiernos sub-estatales en Bruselas es la promover sus prioridades en políticas que les son propias y sobre las que cada vez tiene mayor incidencia la UE (Jefferey 2005).

Sin embargo estas constataciones son insuficientes y es necesario analizar porqué algunos gobiernes tienen un papel más activo que otros. Estamos ante un problema de acción colectiva relativamente tradicional (Olson 1971), puesto que cualquier gobierno territorial europeo puede esperar que otros se organicen, y asuman los costes, y aun así resultar beneficiarios de la atención de la UE a las preocupaciones de dichos gobiernos que se derive del activismo de los demás. Si bien respecto a la primera variable puede darse una explicación reactiva, la organización en red constituye una forma de defender un interés sectorial, siguiendo a Olson (1971) esta explicación sólo resulta útil para un interés claramente identificable y con beneficios individualizados para las regiones que cooperen. Por lo tanto no resulta aplicable para un interés tan vasto como el Arco Atlántico o el Arco Latino.

El papel sobresaliente de Cataluña, Andalucía, País Vasco y la Comunidad Valenciana incitan en un primer momento a buscar explicaciones relativas a la proyección europea de la tensión competencial autonómica - estatal (Marks et al. 1996). Sin embargo en primer lugar llama la atención la ausencia de Galicia y la presencia de actores como Extremadura y Castilla y León. En este sentido parece necesario estudiar factores institucionales relativos al comportamiento de las organizaciones. Para Greenwood (2011a) el activismo regional en redes europeas tiene que ver fundamentalmente con el activismo de su oficina en Bruselas. Aunque podría esperarse que la participación en redes podría ser una forma de coordinar el activismo sin necesidad de una presencia directa, Verboven (2011: 9) señala que disponer de una representación en Bruselas no contradice sino que refuerza la presencia en redes transeuropeas, mientras que las regiones representadas mediante asociaciones nacionales o de grupos de regiones suelen estar menos representadas en dichas redes.

Greenwood (2011a) explica que el mayor o menor grado de participación en redes europeas no expresa tanto una mayor o menor tensión con el gobierno central sino más bien una mayor implicación y experiencia de los responsables de las oficinas de los gobiernos territoriales en distintos proyectos europeos. En este sentido de las 10 organizaciones que participan en mayor número de redes, 9 son CCAA que tienen una sede Bruselas (tabla 5), por lo que es más que posible que la implicación en redes europeas sea en buena medida también el resultado del activismo de dichas oficinas. En lo relativo al nivel local Rodríguez (2011) también confirma que la participación en redes depende fuertemente del grado de implicación personal de los directivos de los Ayuntamientos.

Por último, también resulta notable encontrar una motivación económica, si bien no significa necesariamente que las regiones más prósperas sean aquellas que participan en más redes (Verboven 2011). Aunque los beneficios económicos de la participación en redes suelen ser indirectos, por ejemplo la consecución de una legislación favorable, la participación en redes puede ser importante para desarrollar proyectos susceptibles de financiación europea, puesto que todos estos proyectos requieren que sean promovidos por un grupo de organizaciones de distintos países europeos. Está teniendo lugar un importante cambio por el cual las regiones y entidades locales españoles reciben menos financiación de los programas de cohesión territorial de la UE debido al aumento del PIB 
de España tanto en términos absolutos como en comparación con los nuevos socios comunitarios. Es más que probable que esta tendencia no haga sino incrementarse a partir de 2014 con las nuevas perspectivas financieras de la UE. La Diputación de Barcelona señala que ya "se ha producido una reducción drástica de los recursos que recibe Cataluña y que ha afectado a las entidades locales de la provincia. Ante este escenario, la Dirección de Relaciones Internacionales ha trabajado para buscar nuevas oportunidades que permitieran optimizar de la mejor manera posible el uso de estos fondos y también ha asesorado a los ayuntamientos de la provincia para orientar sus proyectos a las nuevas prioridades que ha marcado la Comisión europea" (Diputación de Barcelona 2011: 11). Rodríguez señala que algunos Ayuntamientos barceloneses ya participan de esta lógica y participan en proyectos en materias de educación, ciudadanía o de naturaleza social (Rodríguez 2011: 10-11). Por lo tanto en el futuro la financiación que se obtenga de la UE para muchos gobiernos autonómicos y locales españoles no será una suerte de "derecho adquirido" en razón de su nivel económico sino que deberá ser obtenido gracias a la promoción de buenas prácticas y políticas innovadoras susceptibles de ser financiados por programas comunitarios distintos de los fondos regionales y de cohesión. Por lo tanto la fuerte presencia de gobiernos territoriales españoles en organizaciones que promueven la innovación, la investigación y el medio ambiente puede explicarse en buena medida por la necesidad de establecer acuerdos con otras entidades locales y regionales para promover proyectos europeos innovadores susceptibles de recibir financiación europea. En este sentido, algunas de estas redes no se dirigen sólo a establecer vínculos con las instituciones comunitarias (Rodríguez 2011: 12) sino a cooperar con otros municipios y regiones europeos.

El estudio ha corroborado las dos hipótesis de partida. En este sentido queda claro que la estrategia de participación en red es una manera de búsqueda de influencia por parte de los gobiernos sub-estatales españoles, puesto que ha quedado acreditado que los mismos participan prioritariamente en redes de defensa de intereses geográficos y económicos. Por otra parte, también se ha mostrado que la participación en redes está relacionada sobre todo con la fuerza de la presencia de las regiones o entidades locales en Bruselas. En este sentido son las regiones mejor representadas en Bruselas y no aquellas que han reducido su presencia o las entidades locales las que participan más activamente en redes europeas. En este sentido, la participación de los gobiernos subestatales en redes europeas se explica como un esfuerzo adicional de las regiones y entidades locales para tratar de defender sus intereses a escala europea por métodos complementarios a los mecanismos formales existentes.

\section{CONCLUSIÓN}

Este artículo ha analizado si los gobiernos sub-estatales españoles se han unido a la tendencia a participar en cada vez más numerosas redes informales señalada por parte de la literatura disponible (Tuñón Navarro 2008b, Greenwood 2011a, Verboven 2011) y cuál ha sido la motivación de dicha tendencia si se hubiera producido.

Se señala en primer lugar que los gobiernos locales y regionales españoles se han unido a esta tendencia si bien de manera desigual. Las CCAA han participado de manera mucho más intensa que sus equivalentes europeos mientras que los entes locales se muestran mucho menos activos que las CCAA, si bien no se encuentran lejos de las regiones europeas. Por lo tanto se puede concluir que como norma general los gobiernos sub-estatales españoles están muy bien representados en estas nuevas formas de cooperación.

Por otra parte se ha señalado que además de ámbitos de cooperación más tradicional, los gobiernos subestatales participan fundamentalmente en redes establecidas en asuntos de su competencia y de defensa de sus intereses territoriales (como por ejemplo promover la integración logística del arco atlántico en redes transeuropeas) y económico (defender al sector vitivinícola o textil). En cuanto a las motivaciones queda claro por lo tanto que la principal es la defender sus prioridades políticas ante una integración europea que impacta cada vez más directamente en sus competencias. Se trata por lo tanto de una actitud proactiva ante la UE. También se ha señalado que hay una importante lógica institucional, es decir, los gobiernos territoriales más activos son por norma general aquellos mejor representados en Bruselas y cuyos representantes mejor conocen las redes y proyectos disponibles (Greenwood 2011a). Por último, la motivación económica puede ser importante en especial para las entidades locales puesto que la participación en redes puede facilitar la participación en proyectos europeos novedosos con otras organizaciones europeas.

Estas conclusiones son relevantes porque señalan un importante cambio de paradigma de los gobiernos subestatales ante la UE. Ante el relativo fracaso de la institucionalización de un papel relevante mediante mecanismos formales como el Comité de las Regiones, estos actores tienden a asociarse y a cooperar para tratar de obtener una influencia directa siguiendo estrategias similares a las de la sociedad civil o los lobbies, es decir, la especialización y la acción colectiva. Pensamos que este cambio de paradigma se enmarca en la tendencia a la politización de la Unión Europea. Si bien los análisis en términos de gobernanza multinivel (Marks et al. 1996) siguen teniendo validez para señalar la participación de distintos actores no estatales en el proceso de decisión, también han recibi- 
do críticas por ofrecer explicaciones en términos de eficiencia más que políticos. En este sentido algunos de estos autores sugieren ahora que es importante tener en cuenta cómo contribuyen los participantes en estos procesos a una mayor politización de la UE (Hooghe y Marks 2009). De este modo la explicación más sencilla de este fenómeno es que ante la complejidad de una situación en la que la UE tiene una influencia directa en sus competencias, los gobiernos sub-estatales se están asociando para tratar de formular sus preferencias políticas en estas materias de manera directa en lugar de confiar en la mediación de sus gobiernos centrales o de otras instituciones.

El análisis de la participación de los gobiernos sub-estatales españoles en redes informales europeas resulta especialmente pertinente en un momento de crisis. En los últimos años ha sido frecuente en la opinión publicada señalar la participación de las CCAA y de los entes locales en asuntos europeos como un ejemplo de gasto público suntuario e incluso absurdo en tiempos de crisis. Este análisis contribuye a explicar porqué a pesar del ruido mediático tan sólo se han cerrado dos de las representaciones regionales menos activas en redes europeas (Asturias y Castilla la Mancha). Contrariamente a los argumentos de esta tendencia crítica de la opinión publicada, se ha señalado que la participación en redes pan-europeas contribuye a aportar una serie de ventajas y beneficios importantes a los entes locales y regionales. Nuestro diagnóstico por lo tanto es que antes que desaparecer, la presencia local y regional en la política comunitaria no puede sino reforzarse por los beneficios que ofrece a los gobiernos que se implican y puesto que otra cosa no dejaría de constituir una dejación de funciones de dichas administraciones ante el impacto de la integración europea en su actividad.

\section{REFERENCIAS}

Asamblea General de Arco Latino (2012) "Manifiesto de Salerno. Un rol renovado para los gobiernos intermedios en Europa: Hacia una mayor Cohesión Territorial en un contexto de Gobernanza Multinivel”, Ravello -Salerno, 16 de marzo de 2012.

Beltrán, Susana (2007) “La cooperación transfronteriza e interterritorial: un clásico renovado” en Revista d'Estudis Autonòmics i Federals, 4, 2007.

Brunazzo, Marco (2010) “Regional Europe”, en: Cini, Michelle y Pérez-Solórzano Borragán, Nieves (eds.): European Union Politics (3 ${ }^{\text {era }}$ edición) Oxford: Oxford University Press, pp. 291-305.

Castellà Andreu, Josep Ma (2008). "Las Comunidades Autónomas en Bruselas: la dimensión externa de la participación autonómica en la Unión Europea" en Revista d'Estudis Autonòmics i Federals, 6, abril 2008, p. 37-91.

De Castro Ruano, José y Ugalde Zubiri, Alexander (2011) Anuario sobre la acción exterior de Euskadi 2008 y 2009, Oñati: Instituto Vasco de Administración Pública. Diputación de Barcelona (2008) Orientacions estratègiques per a l'acció internacional de la Diputació de Barcelona 2008-2011. Barcelona: Diputación de Barcelona Diputación de Barcelona (2011) Memoria de mandat 2007-2011. Direcció de Relacions Internacionals Barcelona: Diputación de Barcelona.

Greenwood, Justin (2011a) "Actors of the common interest? The Brussels Offices of the Regions" Journal of European Integration, Vol. 33, $\mathrm{N}^{\circ} 4$, 2011, pp. 437-451.

Greenwood, Justin (2011b) Interest Representation in the European Union, Basignstoke: Routledge, $3^{\text {rd }}$ edición.

Haas, Peter (1992). "Introduction: Epistemic Communities and International Policy Coordination." International Organization 46 (1).

Hooghe, Liesbet \& Marks, Gary, 2009. "A Postfunctionalist Theory of European Integration: From Permissive Consensus to Constraining Dissensus". British Journal of Political Science, 39(1), pp.1-23.

Jefferey, Charlie (2005) "Regions and the European Union: letting them in and leaving them alone", in S. Wetherill y U. Bernitz (eds.) The role of Regions and subnational actors in Europe (Oxford: Hart), pp. 33-46.

Keating, Michael y Hooghe, Liesbet (2006) "Bypassing the Nation-State? Regions and the EU policy process", en: Richardson, Jeremy (ed.): European Union. Power and policy-making (3era edición) Abingdon: Routledge, pp. 239-255.

Kingdon, John (2003). Agendas, Alternatives, and Public Policies. 2nda edición, New York: Longman.

Knodt, Michèle y Greenwood, Justin (2011) “Territorial and functional interest representation in EU Governance" in Journal of European Integration, 33(4), pp. 349-367.

Marks, Gary et al. (1996) Governance in the European Union, London: Sage.

Morata, Francesc (2002) “Gobernanza multinivel en la Unión Europea” VII Congreso Internacional del CLAD sobre la Reforma del Estado y de la Administración Pública, Lisboa, 8-11 Oct. 2002.

Olson, Mancur (1973) The Logic of Collective Action: Public Goods and the Theory of Groups Cambridge, Massachu- 
setts: Harvard University Press

Quittkat, Christine y Kotzian, Peter (2011). "Lobbying via Consultation - Territorial and Functional Interests in the Commission's Consultation Regime.” Journal of European Integration 33(4): pp. 401-418.

Rodríguez, Josep (2011) "La cooperació transnacional europea dels governs locals: motivacions de les ciutats mitjanes de la província de Barcelona” Working paper Institut Universitari d'Estudis Europeus 32, 2011

Rojo Salgado, Argimiro (1999) “La progresiva regionalización de la Unión Europea”, Revista de Estudios Políticos (Nueva Época), 104, Abril-Junio 1999, pp. 181-211.

Sabatier, Paul A., y Jenkins-Smith, Hank (eds). (1993). Policy Change and Learning: An Advocacy Coalition Approach. Boulder, Colo.: Westview Press.

Sloat, Amanda (2003) “The Preparation of the Governance White Paper” Politics 23(2): pp. 128-136.

Smismans, Stijn (2004) Law, Legitimacy, and European Governance: Functional Participation in Social Regulation. Oxford: Oxford University Press.

Tuñón Navarro, Jorge (2008a) La activación europea de las regiones legislativas: (análisis comparado de las estrategias de Canarias, Escocia, Toscana y Valonia). Madrid: Tesis Doctoral presentada en la Universidad Complutense de Madrid.

Tuñón Navarro, Jorge (2008b) “¿Cómo las regiones influyen en el proceso decisional comunitario? Mecanismos de activación ascendente de las entidades sub-estales europeas” UNISCI Discussion Papers, N 17, pp. 151-172.

Verboven, Tom (2011) “The organizational format of territorial lobbying in Brussels", ponencia preparada para la duodécima conferencia bianual de la European Union Studies Association, Boston, 3-5 de marzo de 2011.

Warleigh, Alex (2002) "The Committee of the Regions", in: Warleigh, Alex (ed.): Understanding European Union institutions. London: Routledge, pp. 177-190

\section{ANEXO I}

\section{TABLA 6}

CCAA incluidas en el estudio

\begin{tabular}{|c|c|}
\hline Comunidad autónoma & Número de redes en que participa \\
\hline Andalucía & 18 \\
\hline Aragón & 12 \\
\hline Asturias & 4 \\
\hline Islas Baleares & 10 \\
\hline País Vasco & 17 \\
\hline Islas Canarias & 6 \\
\hline Cantabria & 5 \\
\hline Castilla La Mancha & 5 \\
\hline Castilla y León & 9 \\
\hline Cataluña & 28 \\
\hline Ceuta & 2 \\
\hline Extremadura & 9 \\
\hline Galicia & 8 \\
\hline La Rioja & 5 \\
\hline Madrid & 7 \\
\hline Melilla & 4 \\
\hline Murcia & 10 \\
\hline Navarra & 8 \\
\hline Valencia & 14 \\
\hline
\end{tabular}


TABLA 7

Diputaciones provinciales y cabildos insulares incluidos en el estudio

\begin{tabular}{|l|l|}
\hline Diputación & Número de redes en que participa \\
\hline Alicante & 1 \\
\hline Almería & 2 \\
\hline Badajoz & 1 \\
\hline Barcelona & 7 \\
\hline Cáceres & 1 \\
\hline Cádiz & 2 \\
\hline Castellón & 2 \\
\hline Gerona & 1 \\
\hline Granada & 1 \\
\hline Diputación foral de Guipúzcoa & 3 \\
\hline Huesca & 1 \\
\hline Jaén & 2 \\
\hline Lérida & 1 \\
\hline Málaga & 2 \\
\hline Consell Insular de Mallorca & 2 \\
\hline Consell Insular de Menorca & 1 \\
\hline Orense & 1 \\
\hline Sevilla & 2 \\
\hline Diputación foral de Vizcaya & 1 \\
\hline Tarragona & 1 \\
\hline Teruel & 2 \\
\hline Zaragoza & 2 \\
\hline
\end{tabular}

TABLA 8

Ayuntamientos incluidos en el estudio

\begin{tabular}{|l|l|}
\hline Ayuntamiento & Número de redes en que participa \\
\hline Barcelona & 10 \\
\hline Hospitalet de Llobregat & 3 \\
\hline Granada & 2 \\
\hline Sevilla & 1 \\
\hline La Coruña & 3 \\
\hline Avilés & 2 \\
\hline San Sebastian & 3 \\
\hline Gijón & 1 \\
\hline Dos Hermanas & 1 \\
\hline Las Palmas & 3 \\
\hline Santiago & 2 \\
\hline Santander & 1 \\
\hline Sevilla & 4 \\
\hline Vigo & 3 \\
\hline Madrid & 3 \\
\hline Sant Boi de Llobregat & 2 \\
\hline
\end{tabular}




\begin{tabular}{|l|l|}
\hline Pamplona & 3 \\
\hline Bilbao & 2 \\
\hline Burgos & 2 \\
\hline Terrassa & 3 \\
\hline Segovia & 3 \\
\hline Baeza & 1 \\
\hline Úbeda & 1 \\
\hline Pozuelo de Alarcón & 2 \\
\hline Zaragoza & 4 \\
\hline Málaga & 2 \\
\hline Vitoria & 1 \\
\hline Prat de Llobregat & 1 \\
\hline Las Rozas & 1 \\
\hline Tarragona & 1 \\
\hline Gerona & 1 \\
\hline Sabadell & 1 \\
\hline Manresa & 2 \\
\hline
\end{tabular}

TABLA 9

Redes incluidas en el studio

\begin{tabular}{|l|l|l|}
\hline Red & & $\begin{array}{l}\text { M ie m b ro s } \\
\text { españoles }\end{array}$ \\
\hline Atlantic Arc & Tema & 19 \\
\hline Intermed & Regiones y ciudades atlánticas & 7 \\
\hline Arco Latino & Dimensión mediterránea de CPRM & 14 \\
\hline Partenalia & Entidades locales mediterráneas & 10 \\
\hline Galicia norte de Portugal & Asuntos sociales e inclusión & 1 \\
\hline ENSA & Cooperación transfronteriza & 4 \\
\hline ELISAN & Asuntos sociales & 1 \\
\hline SIRG & Asuntos sociales & 2 \\
\hline AVEC & Asuntos sociales & 4 \\
\hline Culturelink & Cultura & 2 \\
\hline Europa Nostra & Cultura & 1 \\
\hline Les rencontres & Cultura & 9 \\
\hline Ciudades Educadoras & Cultura & 20 \\
\hline Energy cities in Europe & Cultura educación & 3 \\
\hline Eurolocal Sport & Energía & 5 \\
\hline Climate alliance members & Deporte & 2 \\
\hline Local governments for sustainability & Sostenibilidad & 7 \\
\hline Medcities & Sostenibilidad & 1 \\
\hline Eurotowns & Medio ambiente & 4 \\
\hline ACTE & Ciudades medianas & 6 \\
\hline Euromontana & Sector textil & 4 \\
\hline NECSTouR & Zonas de montaña & 4 \\
\hline & Turismo sostenible & 1 \\
\hline & & \\
\hline
\end{tabular}




\begin{tabular}{|c|c|c|}
\hline GMO-Free & $\begin{array}{l}\text { Contra organismos modificados gené- } \\
\text { ticamente }\end{array}$ & 3 \\
\hline ENRICH & Sanidad & 1 \\
\hline ERISA & Sociedad de la información & 3 \\
\hline EPM & Cooperación transfronteriza & 3 \\
\hline $\mathrm{ACR}+$ & Reciclaje & 10 \\
\hline Nrg4SD & Desarrollo sostenible & 6 \\
\hline ENCORE & Medio ambiente & 3 \\
\hline IMEDOC & Cooperación transfronteriza & 1 \\
\hline AEBR & Cooperación transfronteriza & 8 \\
\hline Wateregio & Uso del agua & 3 \\
\hline CTP - región pirenaica & Cooperación transfronteriza & 4 \\
\hline AREV & Regiones vitícolas & 15 \\
\hline CALRE & Asambleas legislativas & 19 \\
\hline HCN (Health ClusterNET) & Tecnologías sanitarias & 1 \\
\hline METREX & Regiones metropolitanas & 1 \\
\hline MOT & Cooperación transfronteriza & 2 \\
\hline REGLEG & Regiones con poder legislativo & 4 \\
\hline RURACT & Innovación rural & 2 \\
\hline EARLALL & Aprendizaje a lo largo de la vida & 4 \\
\hline EUREGHA & Autoridades sanitarias & 9 \\
\hline POLIS & Transporte & 6 \\
\hline PURPLE & Territorios peri-urbanos & 1 \\
\hline NEREUS & Tecnología espacial & 3 \\
\hline EU 2020 regions & Competitividad & 1 \\
\hline HY-Ramp & Vehículos de hidrógeno y eléctricos & 6 \\
\hline ERLAI & Integración inmigrantes & 7 \\
\hline ECRN & Regiones con industria química & 4 \\
\hline AREFLH & Regiones hortofrutícolas & 6 \\
\hline Four Motors & Producción de automóviles & 1 \\
\hline ERIK & Economía del conocimiento & 9 \\
\hline ERRIN & I+D & 13 \\
\hline AER & Asamblea de regiones & 5 \\
\hline
\end{tabular}

